\title{
Study of Resistance Mechanisms and Evaluation of Biofilm Detection Tests in Clinical Isolates of Pseudomonas aeruginosa Circulating in Yaounde, Cameroon
}

\author{
Yves Le Grand Napa Tchuedji ${ }^{1,2 *}$, Hortense Gonsu Kamga ${ }^{3,4}$, Emilia \\ Lyonga Mbamyah²,3, Anicette Chafa Betbeui ${ }^{3,4}$, Ibrahim Assana Raoul ${ }^{5}$, \\ Giselle Fonyuy Bongnyang ${ }^{6}$, Etienne Guenou ${ }^{2}$ and Francois-Xavier \\ Etoa $^{1}$ \\ ${ }^{1}$ Department of Microbiology, Laboratory of Microbiology, University of Yaoundé I, \\ Cameroon \\ ${ }^{2}$ Department of Public Health, Faculty of Medicine and Biomedical Sciences, \\ University of Yaoundé I, Cameroon \\ ${ }^{3}$ Department of Microbiology, Haematology and Infectious Diseases, Faculty of \\ Medicine and Biomedical Sciences, University of Yaoundé I, Cameroon \\ ${ }^{4}$ Laboratory of Bacteriology, University Teaching Hospital, Cameroon \\ ${ }^{5}$ University and strategic institute of the estuary, Cameroon \\ ${ }^{6}$ General Hospital of Yaoundé, Cameroon \\ *Corresponding Author: Yves Le Grand Napa Tchuedji, Department of Microbiology, \\ Laboratory of Microbiology, University of Yaoundé I, Cameroon.
}

Received: October 20, 2021

Published: November 29, 2021

(C) All rights are reserved by Yves Le Grand Napa Tchuedji., et al.

\section{Abstract}

Background: Antibiotic resistance is a priority problem around the world. The occurrence of SARS-CoV-2 in a context where the resistance of Pseudomonas aeruginosa is increasing, could promote the cohabitation of these microorganisms and an increase in the risk of clinical damage and mortality in infected patients.

Aim: The aim of this study is to determine resistance strategies of clinical isolates of Pseudomonas aeruginosa that circulate in Yaoundé, Cameroon.

Method: A descriptive cross-sectional study was carried out from May 2019 to November 2020. A randomized sampling was conducted. The study of resistance phenotypes was carried out by the disc diffusion method and the double disc synergy method in Mueller-Hinton agar. The study of biofilm formation was performed using the tissue culture plate in Mueller-Hinton broth and Congo red agar.

Results: A total of 30 clinical isolates of Pseudomonas aeruginosa were collected from 300 patients. Pus presented the highest proportion of Pseudomonas aeruginosa followed by urine and blood with frequencies of $60 \%, 16,66 \%$ and $13.33 \%$ respectively. We detected 76,66\% of multi-resistant Pseudomonas aeruginosa with high resistance frequencies to the majority of betalactams and the aminoglycoside, attributed to ertapenem (100\%), cefotaxime (100\%), tircalillin (86.66\%) and tobramycin (60\%). These strains presented $10 \%$ and $3.33 \%$ of carbapenemases and Extended-spectrum beta lactamases (ESBLs) respectively. According to their ability to produce biofilm, $16,66 \%$ and $83,33 \%$ were non-biofilm formers with Plate culture method and culture method on Congo red agar respectively.

Conclusion: This study demonstrated that $P$. aeruginosa strains circulating in the Center region have several enzymatic mechanisms of resistance to antibiotics associated with a high production of biofilm. The study of biofilm formation shows a significant difference between the tissue culture technique and the Congo red agar culture technique.

Keywords: Pseudomonas aeruginosa; Resistance Phenotypes; Carbapenemases; ESBLs; Resistance Genes 


\section{Abbreviations}

ESBLs: Extended-spectrum Beta Lactamases; P. aeruginosa : Pseudomonas aeruginosa; SARS-CoV-2: Severe Acute Respiratory Syndrome Coronavirus 2; MAR: Multiple Antibiotic Resistance; CASFM: Antibiogram Committee of the French Microbiology Society; OD: Optical Density

\section{Introduction}

In the past, multi-resistant bacteria were first associated with nosocomial infections, but today they cause several infections in the community, causing considerable economic losses. Antibiotic resistance is present in all countries with an increased risk of clinical damage and mortality in patients exposed to resistant bacteria [1]. This situation is still very worrying in Africa, where several factors contribute to the emergence and transmission of resistant strains. Pseudomonas aeruginosa is known to be one of the major causes of opportunistic infections in immunocompromised patients associated with high mortality. In the world, and particularly in Cameroon, the situation of antimicrobial resistance is even more worrying, this can be attributed to reckless prescription of antibiotics by unqualified personnel, self-medication, the widespread use of counterfeit drugs whose dosage in active compounds is unknown, poor sanitation and hygiene in medical facilities and usage in subtherapeutic doses [2]. Many studies have shown that subminimal inhibitory concentrations of antibiotics could induce mutagenesis in clinical isolates of Pseudomonas aeruginosa [3].

P. aeruginosa is one of the multi-resistant bacteria which possess several virulence factors and which infect the respiratory tract. The occurrence of SARS-CoV-2 in a context where resistance to antibiotics is increasing, could promote the coexistence of these microorganisms in the respiratory tract. This coinfection can cause an amplification of the physiopathological mechanisms and accelerate the clinical and functional degradation of patients carrying SARS-CoV-2.

Very little data are available on the exhaustive list of the different strategies of resistance found in Pseudomonas aeruginosa in the Cameroonian population. This implies a surveillance of resistance focused on finding mechanisms of resistance of $P$. aeruginosa circulating in Cameroon. The aim of this study is to determine resistance strategies of Pseudomonas aeruginosa in Yaounde.

\section{Methods}

Isolation and handling of bacterial strains

The isolates of Pseudomonas aeruginosa from 300 patients attending the University Teaching Hospital of Yaounde, the Central Hospital of Yaounde, the General Hospital, and the Centre Pasteur of Cameroon were collected between May 2019 and October 2020. Bacteria isolates were conserved in Brain Hearth infusion broth supplemented with glycerol (10\%) and transported in ice to the bacteriology laboratory of the University Teaching Hospital.

\section{Identification}

Presumptive identification of the isolates was performed in each collection site using the catalase, oxidase, mannitol and citrate Simmons agar test. The isolates were revived on Mueller-Hinton agar and further identified using the Hajna Kliger media to assess glucose, lactose fermentation and API 20 NE (BioMérieux, France) following the manufacturer's instructions.

\section{Antimicrobial susceptibility testing}

Antimicrobial Susceptibility tests were conducted according to the recommendations of the Antibiogram committee of the French Microbiology Society using the disc diffusion method on MuellerHinton agar (CASFM) [4]. The bacterial inoculum adjusted to 0.5 Mc Farland was inoculated on Mueller-Hinton agar. Fifteen minutes later, thirteen antibiotic discs were deposited on the Mueller-Hinton agar. The following discs were used: tircacillin $(75 \mu \mathrm{g})$, tircacillin/clavulanic acid (85 $\mu \mathrm{g})$, piperacillin (30 $\mu \mathrm{g})$, piperacillin/ tazobactam (36 $\mu \mathrm{g})$, ceftazidim $(30 \mu \mathrm{g})$, cefotaxim (30 $\mu \mathrm{g})$, imipenem $(10 \mu \mathrm{g})$, meropenem $(10 \mu \mathrm{g})$, ertapenem $(10 \mu \mathrm{g})$, tobramycin $(10 \mu \mathrm{g})$, ciprofloxacin $(5 \mu \mathrm{g})$, netilmicin $(30 \mu \mathrm{g})$, norfloxacin (10 $\mu \mathrm{g})$. This was left at room temperature for 15 minutes and then incubated at $37^{\circ} \mathrm{C}$ for 24 hours. Inhibition zones were measured after incubation and scored as susceptible, intermediate or resistant. The multiple antibiotic resistance (MAR) index was determined by dividing the total number of resistances to antimicrobials for each isolate to the total number of tested antimicrobials.

\section{Detection of extended-spectrum beta lactamases}

Extended-spectrum beta lactamases (ESBLs) are enzymes found in certain bacteria and are responsible for their resistance to antibiotics such as penicillins and cephalosporins. The double disc 
synergy was used to screen all the isolates for ESBLs production as recommended by CASFM [4]. Antibiotic discs ceftazidime, cefotaxime, cefepime were placed $30 \mathrm{~mm}$ around ticarcillin/ clavulanic acid disc in pre-inoculated Mueller Hinton agar. The plates were incubated aerobically at $37 \circ \mathrm{C}$ for $24 \mathrm{~h}$. The production of heartshaped clear zones that distorts the zone of inhibition indicates positive results for ESBLs production.

\section{Detection of carbapenemase}

The search for carbapenemases was carried out by the disc diffusion method according to the recommendation of CASFM [4]. The bacterial inoculum prepared according to 0.5 McFarland standard was inoculated on Mueller-Hinton Agar. After 15 minutes, imipenem disc $(10 \mu \mathrm{g})$ was deposited on the plate. The whole is left to prediffused for 15 minutes and then incubated aerobically for 24 hours at $37^{\circ} \mathrm{C}$. The observation of inhibition zones less than $28 \mathrm{~mm}$ testifies to a presence of carbapenemases.

\section{Detection of cephalosporinases}

The search for cephalosporinases was carried out by the disc diffusion test on solid medium according to the recommendation of CASFM [4].

The bacterial inoculum prepared according to 0.5 McFarland standard was inoculated on Mueller-Hinton agar. After 15 minutes cephalosporin discs, tircacillin, tircacillin/clavulanic acid was deposited on the surface of the agar plate. The whole is left prediffused for 15 minutes and then incubated aerobically for 24 hours at $37^{\circ} \mathrm{C}$. The observation of resistance to these antibiotics confirm the presence of cephalosporinases.

Determination of biofilm formation by tissue culture plate method

The study of biofilm formation was carried out according to the method described by 0'Toole., et al. [5]. To perform this, $150 \mu \mathrm{L}$ of brain heart infusion broth was added to each well of a sterile microtitre plates. Then $10 \mu \mathrm{L}$ of inoculum adjusted to $0.5 \mathrm{McFar}-$ land was inoculated into each well. Negative and positive controls were included, containing $150 \mu \mathrm{L}$ of brain heart infusion broth and $150 \mu \mathrm{L}$ of brain heart infusion broth containing strains producing biofilm respectively. The whole was incubated for 48 hours at $37^{\circ}$ C. After incubation, each well was emptied gently using a syringe, avoiding contact with the wall of the well. The wells were then wa- shed 3 times with distilled water and then stained with $1 \%$ crystal violet for 5 minutes. Then the wells were washed twice and then dried. Ethanol-acetone (75:25) was pipetted into each well in order to detach any biofilm which is on the wall of the well and the optical density read at $570 \mathrm{~nm}$. The micro-well plates were agitated for one minute prior to measuring the turbidity. The tests were repeated three times. The categorization of isolates was done according to the recommendations of Christensen., et al. [6], which states: $\mathrm{OD} \leq \mathrm{OD}_{\mathrm{t}}$ : not producing biofilm; $\mathrm{OD}_{\mathrm{t}} \times 2 \leq \mathrm{OD}<\mathrm{OD}_{\mathrm{t}} \times 4$ : moderate production of biofilm; $\mathrm{OD}_{\mathrm{t}} \times 4 \leq \mathrm{OD}$ : highly biofilm producer.

Determination of biofilm formation by culture on Congo red agar method

The study of biofilm formation was carried out according to the method described by Hou., et al. [7]. For this, the pure colonies of Pseudomonas aeruginosa were inoculated on Congo red agar supplemented with saccharose $10 \mathrm{~g} / \mathrm{L}$. The Petri dishes were then incubated at $37^{\circ} \mathrm{C}$ for $24-48$ hours. After incubation the presence of strains producing slimes give rough black colonies, non-producing strains give red colonies with a smooth surface, and strains with varying phenotypes give colonies with a red outline and black center [8].

\section{Results}

\section{Frequency of Pseudomonas aeruginosa}

Samples were taken from 300 patients. Thirty cultures were positive for Pseudomonas aeruginosa (10\%). Among the 30 strains, 13 were isolated at the Centre Pasteur of Cameroon and 17 in hospital with $76.47 \%$ of hospitalized patient. Figure 1 shows the proportion of infected patients according to the unit of origin.

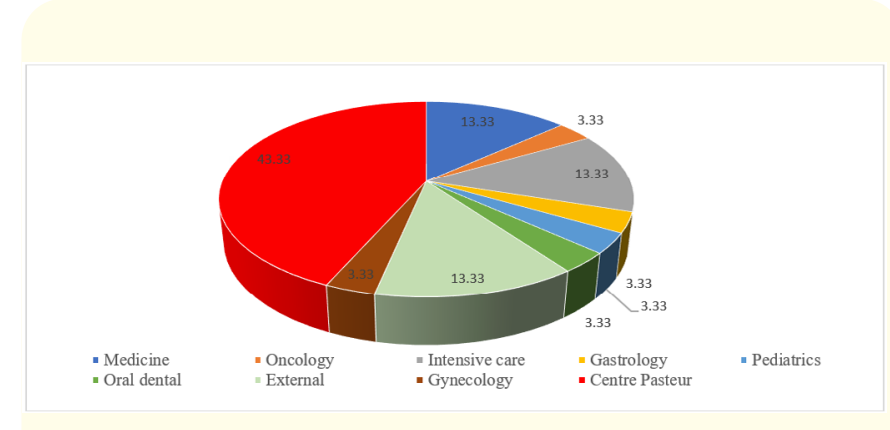

Figure 1: Distribution of infected patients according to the unit of origin. 
As shown by figure 1, the Centre Pasteur of Cameroon have the highest frequency of Pseudomonas aeruginosa followed by the unit of medicine and intensive care with a frequency of $43.33 \%, 13.33 \%$ and $13.33 \%$ respectively. The strains of Pseudomonas aeruginosa were isolated from different samples (Table 1).

\begin{tabular}{|c|c|c|c|c|c|c|c|}
\hline & Urine & Wound & Bedsore & Blood & Lochia & Pus & Total \\
\hline $\mathrm{N}_{0}$ positive $(\%)$ & $5(16,66)$ & $1(3,33)$ & $1(3,33)$ & $4(13,33)$ & $1(3,33)$ & $18(60)$ & $30(100)$ \\
\hline
\end{tabular}

Table 1: Frequency of Pseudomonas aeruginosa by sample.

Pus presented the highest proportion of Pseudomonas aeruginosa $(60 \%)$ followed by urine $(16,66 \%)$, blood $(13,33 \%)$ wounds (3.33\%) bedsores (3.33\%) and lochia (3.33\%).

Antibiotic susceptibility profile

The susceptibility profile (Figure 2 ) revealed a high resistance to the majority of betalactams and the aminoglycoside, attributed to ertapenem (100\%), cefotaxime (100\%), tircacillin $(86.66 \%)$ and tobramycin (60\%). We also noted resistance to netilmicin, imipenem and meropenem with respective frequency of $26.66 \%$, $10 \%$ and $20 \%$. High resistance to beta-lactam inhibitors was observed for piperacillin/tazobactam and tircacillin/clavulanic acid with frequencies of 86.66 and 50\% respectively. Resistance to quinolones varying from 36.66 for norfloxacin to $40 \%$ for ciprofloxacin.

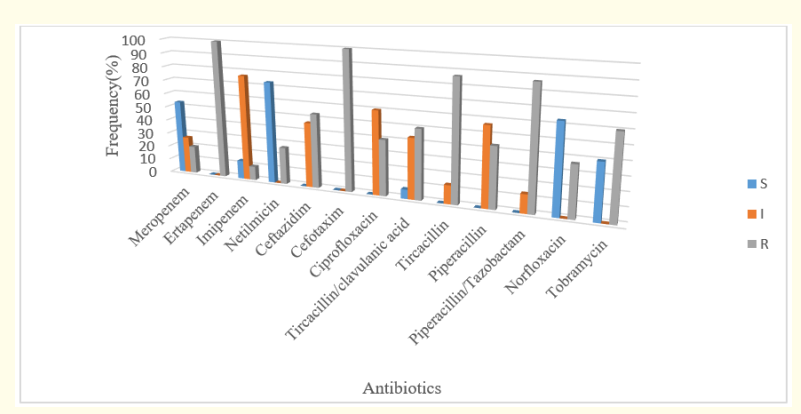

Figure 2: Antibiotic susceptibility profile of Pseudomonas aeruginosa.

R: Resistant; I: Intermediate; S: Susceptible.

Among the 30 strains collected, $76.66 \%$ were multidrug resistant. The determination of the multi-resistance index did not consider natural resistance. Table 2 shows the frequency of Pseudomonas aeruginosa according to the multi-resistance index.
The multi-resistance index varied from 0.23 to 0.84 with $39.11 \%$ of the micro-organisms which having a multi-resistance index greater than 0.50 .

The distribution of Pseudomonas aeruginosa according to age group (Figure 3) shows a high frequency of multi-resistance in each group.

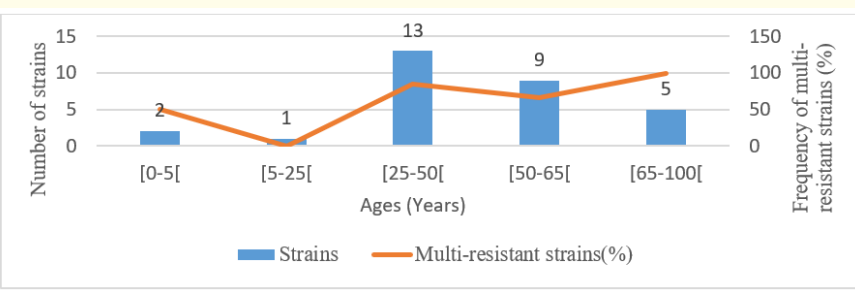

Figure 3: Frequency of Pseudomonas aeruginosa isolates according to age and multi-resistance.

Patients aged between [25-50[ years and [50-65[ years were the most infected. However, a high proportion of multi-resistant bacteria was observed between the ages of [25-50[, [50-65[ and [65-100[ years although the age group of [65-100[ years had the highest proportion of multi-resistant bacteria.

\section{Resistance phenotypes of Pseudomonas aeruginosa}

Table 3 presents the resistance phenotypes detected in this study. The wild phenotypes were conserved in all the isolates. However, we detected the presence of ESBLs and carbapenemases with respective frequencies of $3.33 \%$ and $10 \%$. No positive result was found for cephalosporinases.

\section{Study of biofilm formation by Pseudomonas aeruginosa}

The study of biofilm formation was carried out using two methods described in previous studies. The results obtained by these 


\begin{tabular}{|l|c|c|c|c|}
\hline & Wild & ESBLs & $\begin{array}{c}\text { Carbapen- } \\
\text { emases }\end{array}$ & $\begin{array}{c}\text { Cephalo- } \\
\text { sporinases }\end{array}$ \\
\hline Frequency $\mathrm{n}(\%)$ & 100 & 3,33 & 10 & 0 \\
\hline
\end{tabular}

two techniques show a difference in frequency of the strains producing biofilms. Table 4 shows the frequency of Pseudomonas aeruginosa according to the plate culture technique and the Congo red agar culture technique.

Table 3: Resistance phenotypes of Pseudomonas aeruginosa.

\begin{tabular}{|c|c|c|c|c|c|c|}
\hline \multicolumn{3}{|c|}{ Plate culture method } & \multicolumn{3}{c|}{ Congo Red Agar culture method } \\
\hline Biofilm formation & $\begin{array}{c}\text { Strong bio- } \\
\text { film formers }\end{array}$ & $\begin{array}{c}\text { Moderate } \\
\text { biofilm formers }\end{array}$ & $\begin{array}{c}\text { Non-biofilm } \\
\text { formers }\end{array}$ & $\begin{array}{c}\text { biofilm } \\
\text { formers }\end{array}$ & $\begin{array}{c}\text { Variable biofilm } \\
\text { formers }\end{array}$ & $\begin{array}{c}\text { Non-biofilm } \\
\text { formers }\end{array}$ \\
\hline Frequency n (\%) & $9(30 \%)$ & $16(53,33 \%)$ & $5(16,66 \%)$ & $3(10 \%)$ & $2(6,66 \%)$ & $25(83,33 \%)$ \\
\hline
\end{tabular}

Table 4: Frequency of Pseudomonas aeruginosa isolates according to their ability to produce Biofilm.

Thirty percent of the isolates of Pseudomonas aeruginosa were strong producers of biofilms and $53.33 \%$ carried out a moderate production of biofilm with plate culture technique, while $10 \%$ and $6,66 \%$ where biofilm formers and variable biofilm formers after culture on Congo red agar. The production of biofilm is one of the resistance strategies used by Pseudomonas aeruginosa to resist antibiotics. However, 16,66\% and 83, 33\% were non-biofilm formers with Plate culture method and culture method on Congo red agar respectively. This difference $(\mathrm{P}<0.05)$ observed between the two techniques on the frequency of bacteria which do not produce biofilms is statistically significant.

\section{Discussion}

Among the 300 patients sampled, 10\% were infected with Pseudomonas aeruginosa. These results are similar to those of Barbier and Wolff [9], in France who found a frequency of $10 \%$ and lower to that of Clotilde., et al. [10], who found a frequency of $15.4 \%$ in the city of Douala. This city is the economic capital of Cameroon where the majority of commercial and industrial activities are carried out leading to overcrowding of the city, unsanitary environment and non-observance of hygiene measure [11]. The Centre Pasteur of Cameroon have the highest frequency of Pseudomonas aeruginosa. This could be explained by the fact that this laboratory receives the majority of patients compared to laboratories of hospitals.

Concerning unit of origin in hospitals, medicine and intensive care unit had the highest frequency of Pseudomonas aeruginosa. Many studies show that medicine and intensive care have a high proportion of infection compared to other units. The work carried out by Gonsu., et al. [12], also revealed that medicine and intensive care units had the highest proportion of infections compared to pediatrics, surgery, neonatalogy and ophtamology. Pus presented the highest proportion of Pseudomonas aeruginosa (60\%) followed by urine $(16,66 \%)$ and blood $(13,33 \%)$. These results are different from those of Ndip., et al. (2005) who found a high prevalence of P. aeruginosa in urine (30\%) and wounds (28\%) compared to pus (16\%). This could be explained by its ubiquitous nature.

A high resistance to the majority of betalactams was observed with a frequency of $100 \%$ for ertapenem, $100 \%$ for cefotaxime, $86.66 \%$ for tircacillin and $60 \%$ for tobramycin. This family of drugs has been affected by the constant increase in bacteria resistance [13]. We also noted $10 \%$ and $20 \%$ of resistance to imipenem and meropenem respectively. Carbapenem-resistant $P$. aeruginosa is one of the resistant ESKAPE pathogens in which there is a critical need for the development of new antibiotics to treat infections [14]. Resistance was noted to norfloxacin and ciprofloxacin with a frequency of $36,66 \%$ and $40 \%$ respectively. This bacteria has developed and maintained resistance to quinolones for many years [15]. The highest proportion of multi-resistant strains was observed in the age group of [65-100 [ years. This could be explained by the immune failure in elderly patients which promotes the growth of bacteria and gives them time to develop resistance mechanisms.

We detected $10 \%$ of carbapenemase and 3,33\% of ESBLs. The study of Madaha., et al. [16], shows that the double disc synergy test have a low capacity to detect ESBLs on Pseudomonas aeruginosa compared to the combined disc method. This constitute a limit 
in the detection of resistance mechanisms because of the permanent unavailability of the antibiotic combined disc in developing country as Cameroon.

The difference observed between plate culture method and culture method on Congo red agar on the frequency of bactaria which do not biofilms is statistically significant. The work carried out by Madaha., et al. [16], revealed that under the same temperature, only $17.33 \%$ of isolates of Pseudomonas aeruginosa did not produce biofilms with plate culture method while the work of Kengne., et al. [17], showed a proportion of $42.85 \%$ of Pseudomonas aeruginosa strains which did not produce biofilm with the Congo red agar culture technique. This confirms the low proportion of biofilm detection by the Congo red agar culture method. It is therefore important to determine the performance of these tests in order to make an optimal choice for the detection of biofilm formation.

\section{Ethical Approval}

This study was approved by the Ethical committee of the delegation of public health for the center Region of Cameroon under the approval number 0191AAR/MINSANTE/DRSPL/BCASS. Anonymity of participants and confidentiality of results were scrupulously respected.

\section{Conclusion}

This study presented many resistance mechanisms of Pseudomonas aeruginosa circulating in Yaoundé Cameroon, such as enzymes that inactivate antibiotics and the development of biofilms. The Carbapenemases were the most represented. The difference observed between the tests for the detection of biofilm, proves a need to determine the performances, such as sensitivity and the specificity to make an optimal choice of these tests.

\section{Acknowledgment}

We thanks Rosanne NGOME who participated in the collection of strains at the Centre Pasteur of Cameroon, Michelle DJUIDJE, Melissa TSANGA and Ornella MINLONG who participated in the collection of strains at the Central Hospital of Yaoundé.

\section{Bibliography}

1. Battraud Paul. "La résistance aux antibiotiques, un mythe ou une réalité?" Thèse pour le diplome d'etat de docteur en pharmacie (2017): 1-128.
2. Nko'o Amvene Samuel and Marie Claire Okomo Assoumou. "Antibiotiques et résistance bactérienne : un sujet de préoccupation". Health Sciences and Disease 14.4 (2013): 1-2.

3. Valencia Estela Ynés., et al. "Ciprofloxacin-mediated mutagenesis is suppressed by subinhibitory concentrations of amikacin in Pseudomonas aeruginosa". Antimicrobial Agents and Chemotherapy 61.3 (2017): e2107-e2116.

4. CA-SFM. Recommendations 1 (2020): 1-9.

5. O'Toole., et al. "Flagellar and twitching motility are necessary for Pseudomonas aeruginosa biofilm development". Molecular Microbiology 30.2 (1998): 295-304.

6. Christensen., et al. "Adherence of coagulase-negative staphylococci to plastic tissue culture plates: a quantitative model for the adherence of staphylococci to medical devices". Journal of Clinical Microbiology 22.6 (1985): 996-1006.

7. Wenbo Hou., et al. "The Association for Research in Vision and Ophthalmology". Investigative Ophthalmology and Visual Science 53.9 (2012): 5624-5631.

8. Mathur., et al. "Detection of biofilm formation among the clinical isolates of Staphylococci: An evaluation of three different screening methods". Indian Journal of Medical Microbiology 24.1 (2006): 25-29.

9. François Barbier and Michel Wolff. "Multirésistance chez Pseudomonas aeruginosa Vers l'impasse thérapeutique?" Medicine/Sciences 26.11 (2010): 960-968.

10. Njall, pouth Clotilde., et al. "Écologie bactérienne de l'infection nosocomiale au service de réanimation de l'hôpital Laquintinie de Douala, Cameroun". Pan African Medical Journal 14.140 (2013): 1-8.

11. NAPA TCHUEDJI., et al. "Epidemiology of Pseudomonas aeruginosa and Its Resistance in Cameroon: Systematic Review and Meta Analysis". Global Advanced Research Journal of Microbiology 9.5 (2020): 069-072.

12. Gonsu Kamga., et al. "Caractérisation phénotypique des souches de Pseudomonas aeruginosa isolées dans la ville de Yaoundé (Cameroun)". African Journal of Pathology and Microbiology 4 (2015): 1-4.

13. Worthington Roberta and Melander Christian. "Overcoming Resistance to $\beta$-Lactam Antibiotics". Journal of Organic Chemistry 78.9 (2013): 4207-4213.

14. Founou Raspail Carrel., et al. "Clinical and economic impact of antibiotic resistance in developing countries: A systematic review and meta-analysis". Plos One 12.12 (2017): e0189621. 
15. Robert Hancock Rew and Speert David. "Antibiotic resistance in Pseudomonas aeruginosa: mechanisms and impact on treatment". Drug Resistant Update 3.4 (2000): 247-255.

16. Madaha Estelle Longla., et al. "Occurrence of blaTEM and blaCTXM Genes and Biofilm-Forming Ability among Clinical Isolates of Pseudomonas aeruginosa and Acinetobacter baumannii in Yaoundé, Cameroon". Microorganisms 8.708 (2020): 1-22.

17. Kengne Landry. "Contribution à la lutte contre les contaminants microbiens des locaux et des collections des bibliothèques au moyen d'huiles essentielles". Thèse de Doctorat. Université de Yaoundé I. Cameroun (2019): 1-236.

Volume 4 Issue 12 December 2021

(C) All rights are reserved by Yves Le Grand Napa

Tchuedji., et al. 